HD-THEP-99-38

\title{
Gauge-invariant nonlocal quark condensates in QCD: a new interpretation of the lattice results
}

\author{
E. Meggiolaro ${ }^{\mathrm{a}}$ \\ anstitut für Theoretische Physik, Universität Heidelberg, Philosophenweg 16, D-69120 Heidelberg, \\ Germany
}

\begin{abstract}
We study the asymptotic short-distance behaviour as well as the asymptotic large-distance behaviour of the gauge-invariant quark-antiquark nonlocal condensates in QCD. A comparison of some analytical results with the available lattice data is performed.
\end{abstract}

\section{INTRODUCTION}

In a recent paper [1] we have presented a lattice determination of the quark-antiquark nonlocal condensates, defined as:

$C_{i}(x)=-\sum_{f=1}^{4}\left\langle\operatorname{Tr}\left[\bar{q}_{a}^{f}(0)\left(\Gamma^{i}\right)_{a b} S(0, x) q_{b}^{f}(x)\right]\right\rangle$,

where $S(0, x)$ is the Schwinger line needed to make $C_{i}(x)$ gauge-invariant and $\Gamma^{i}$ are the sixteen independent matrices of the Clifford's algebra acting on the Dirac indices $a, b$. The trace in (1) is taken with respect to the colour indices.

Making use of T,P invariance one can prove that all the correlators (1) vanish, except those with $\Gamma^{i}=\mathbf{1}$ ("scalar" nonlocal condensate) and with $\Gamma^{i}=\gamma_{E}^{\mu}$ and $\mu$ in the direction of $x$ ("longitudinal-vector" nonlocal condensate):

$$
\begin{array}{r}
C_{0}(|x|)=-\sum_{f=1}^{4}\left\langle\operatorname{Tr}\left[\bar{q}_{a}^{f}(0) S(0, x) q_{a}^{f}(x)\right]\right\rangle \\
C_{v}(|x|)=-\frac{x_{\mu}}{|x|} \sum_{f=1}^{4}\left\langle\operatorname{Tr}\left[\bar{q}_{a}^{f}(0)\left(\gamma_{E}^{\mu}\right)_{a b} S q_{b}^{f}(x)\right]\right\rangle .
\end{array}
$$

These two quantities play a relevant role in many applications of QCD sum rules, especially for studying the meson form factors and the meson wave functions [2 5]. The nonlocal quark condensates have been also determined within the single instanton approximation of the instanton liquid model [6].

The lattice computations of Ref. [1 have been performed both in the quenched approximation and in full QCD using four degenerate flavours of staggered fermions [whence the sum over the flavour index $f$ in (1)] and the $S U(3)$ Wilson action for the pure-gauge sector.

In full QCD the nonlocal condensates have been measured on a $16^{3} \times 24$ lattice at $\beta=$ 5.35 and two different values of the quark mass: $a \cdot m_{q}=0.01$ and $a \cdot m_{q}=0.02$ ( $a$ being the lattice spacing). For the quenched case the measurements have been performed on a $16^{4}$ lattice at $\beta=6.00$, using valence quark masses $a \cdot m_{q}=0.01,0.05,0.10$, and at $\beta=5.91$ with a quark mass $a \cdot m_{q}=0.02$. Further details, as well as a remark about the reliability of the results obtained for the longitudinal-vector nonlocal condensate, can be found in [1].

In what follows we shall concentrate on the scalar nonlocal condensate $C_{0}(x)$. In Ref. [1] a best fit to the data with the following function has been tried:

$C_{0}(x)=A_{0} \exp \left(-\mu_{0} x\right)+\frac{B_{0}}{x^{2}}$.

The form of the perturbative-like term $B_{0} / x^{2}$ is that obtained in the leading order in perturbation theory, in the chiral limit $m_{q} \rightarrow 0$.

The quantity of greatest physical interest which can be extracted from our lattice determinations is the correlation length $\lambda_{0} \equiv 1 / \mu_{0}$ of the scalar quark-antiquark nonlocal condensate. At the lightest quark mass $a \cdot m_{q}=0.01$ we have obtained the value $\lambda_{0} \simeq 0.63 \mathrm{fm}$ [1].

Here we study the asymptotic short-distance behaviour as well as the asymptotic large- 
distance behaviour of the gauge-invariant quarkantiquark nonlocal condensates in QCD. The large-distance behaviour is derived by making use of a relation of these correlators to the twopoint functions for the scalar and pseudoscalar $\bar{Q} q$ meson operators in the limit of the heavy-quark mass $M_{Q} \rightarrow \infty$. A comparison of some analytical results with the available lattice data will be performed.

\section{SHORT-DISTANCE BEHAVIOUR}

The short-distance behaviour of the correlators is described by an "operator product expansion" (O.P.E.), having the form:

$C_{0}(x)=\frac{\tilde{B}_{0}}{x^{2}}-\langle: \bar{q} q:\rangle+\ldots$

The vacuum expectation values of the local operators, such as $\langle: \bar{q} q:\rangle$, appear as expansion coefficients of the nonlocal condensate $C_{0}(x)$ in a Taylor series in the variable $x^{2}$. In the literature 2 4 the nonperturbative part of the scalar nonlocal condensate is often parametrized with a Gaussian function:

$C_{0}^{(n . p .)}(x)=\tilde{A}_{0} \exp \left(-\frac{1}{8} \mu_{q}^{2} x^{2}\right)$.

The parameter $\mu_{q}^{2}$ characterizes the nonlocality of the quark condensate and it is given by 2 ,

$\mu_{q}^{2}=\frac{\left\langle: \bar{q} D^{2} q:\right\rangle}{\langle: \bar{q} q:\rangle}$,

where $D_{\mu}=\partial_{\mu}+i g A_{\mu}^{a} T^{a}$ is the covariant derivative. By the equations of motion in the chiral limit, the parameter $\mu_{q}^{2}$ is also related to the mixed quark-gluon condensate:

$\mu_{q}^{2}=\frac{\left\langle: \bar{q}\left(i g \sigma_{\mu \nu} G_{\mu \nu}^{a} T^{a}\right) q:\right\rangle}{2\langle: \bar{q} q:\rangle}$.

From the QCD sum-rules phenomenology one finds the following estimate for $\mu_{q}^{2}$ [7]:

$\mu_{q}^{2}=0.50(5) \mathrm{GeV}^{2}$.

Therefore we have also tried a best fit to the data of the scalar nonlocal condensate with the function

$C_{0}(x)=\tilde{A}_{0} \exp \left(-\frac{1}{8} \mu_{q}^{2} x^{2}\right)+\frac{\tilde{B}_{0}}{x^{2}}$.
It comes out that the data are well fitted by this function [even if the $\chi^{2} / N_{\text {d.o.f. }}$ is slightly larger than in the exponential case, Eq. (3)]. The following value for $\mu_{q}^{2}$ is obtained from the best fit to the full-QCD data at $\beta=5.35$ and $a \cdot m_{q}=0.01$ :

$\mu_{q}^{2}=0.46(5) \mathrm{GeV}^{2}$.

This value is in good agreement with the QCD sum-rule estimate (8).

\section{LARGE-DISTANCE BEHAVIOUR}

In this section we study the asymptotic largedistance behaviour of the quark correlators. The starting point is the fermion propagator in an external gluon field $A_{\mu}=A_{\mu}^{a} T^{a}$, in the infinite mass limit $M_{Q} \rightarrow \infty$ (static fermion limit) [8,9]:

$$
\begin{aligned}
& \left\langle Q_{a, \alpha}(z) \bar{Q}_{b, \beta}\left(z^{\prime}\right)\right\rangle_{(A)}= \\
& \quad=\delta^{(3)}\left(\vec{z}-\vec{z}^{\prime}\right)\left[\mathrm{P} \exp \left(i g \int_{z_{0}}^{z_{0}^{\prime}} \mathrm{d} t A_{0}(\vec{z}, t)\right)\right]_{\alpha \beta} \\
& \quad \times\left[\theta\left(z_{0}-z_{0}^{\prime}\right)\left(\mathrm{P}_{+}\right)_{a b} \mathrm{e}^{-i M_{Q}\left(z_{0}-z_{0}^{\prime}\right)}\right. \\
& \left.\quad+\theta\left(z_{0}^{\prime}-z_{0}\right)\left(\mathrm{P}_{-}\right)_{a b} \mathrm{e}^{i M_{Q}\left(z_{0}-z_{0}^{\prime}\right)}\right]
\end{aligned}
$$

where $\alpha, \beta=1, \ldots, N_{c}$ are colour indices, $a, b$ are Dirac indices and

$\mathrm{P}_{+} \equiv \frac{\mathbf{1}+\gamma^{0}}{2} \quad ; \quad \mathrm{P}_{-} \equiv \frac{\mathbf{1}-\gamma^{0}}{2}$.

Let us consider now the following mesonic correlators:

$$
\begin{gathered}
M_{S}^{(Q)}\left(x^{0}\right) \equiv \sum_{f=1}^{4} \int \mathrm{d}^{3} \vec{z}^{\prime}\left\langle O_{S}^{f \dagger}\left(x^{0}, \vec{z}^{\prime}\right) O_{S}^{f}(0)\right\rangle \\
M_{P S}^{(Q)}\left(x^{0}\right) \equiv \sum_{f=1}^{4} \int \mathrm{d}^{3} \vec{z}^{\prime}\left\langle O_{P S}^{f \dagger}\left(x^{0}, \vec{z}^{\prime}\right) O_{P S}^{f}(0)\right\rangle
\end{gathered}
$$

where $x^{0}>0$ and the mesonic operators $O_{S}^{f}$ and $O_{P S}^{f}$ are so defined:

$O_{S}^{f} \equiv \bar{q}^{f} \gamma^{0} Q \quad ; \quad O_{P S}^{f} \equiv \bar{q}^{f} \gamma^{0} \gamma^{5} Q$

The spin and colour indices are contracted. In other words, $O_{S}^{f}$ is the time component of the vector current $J_{f}^{\mu}=\bar{q}^{f} \gamma^{\mu} Q$, while $O_{P S}^{f}$ is the time component of the axial-vector current $J_{5, f}^{\mu}=$ 
$\bar{q}^{f} \gamma^{\mu} \gamma^{5} Q$ : we call $O_{S}^{f}$ "scalar" meson operator and $O_{P S}^{f}$ "pseudoscalar" meson operator. Using Eq. (11) and performing the analytic continuation from Minkowskian to Euclidean space $\left(x^{0} \rightarrow-i x_{4}\right.$, with $\left.x^{0}, x_{4}>0\right)$, one easily finds the following relations:

$M_{E, P S}^{(Q)}\left(x_{4}\right)-M_{E, S}^{(Q)}\left(x_{4}\right)=\mathrm{e}^{-M_{Q} x_{4}} C_{0}\left(x_{4}\right) ;$

$M_{E, P S}^{(Q)}\left(x_{4}\right)+M_{E, S}^{(Q)}\left(x_{4}\right)=\mathrm{e}^{-M_{Q} x_{4}} C_{v}\left(x_{4}\right)$.

$C_{0}\left(x_{4}\right)$ and $C_{v}\left(x_{4}\right)$ are precisely the quark correlators measured on the lattice, defined by Eqs. (2). As a candidate for the heavy quark $Q$, we can take the $b$ (bottom) quark, for which we know the pseudoscalar mesons of the type $I\left(J^{P}\right)=\frac{1}{2}\left(0^{-}\right)$, with mass $M_{B} \simeq 5.3 \mathrm{GeV}$. We now consider the Eqs. (15) in the asymptotic limit $x_{4} \rightarrow \infty$. If we make the assumption (supported by the experimental evidence) that the lightest $B$-mesons are the pseudoscalar ones, we find that:

$C_{0, v}\left(x_{4}\right) \underset{x_{4} \rightarrow \infty}{\sim} A_{\infty} \mathrm{e}^{-\mu_{\infty} x_{4}}$,

where

$A_{\infty}=2 F_{B}^{2} M_{B} \quad ; \quad \mu_{\infty}=M_{B}-M_{b}$.

$M_{b}$ is the mass of the $b$ quark. $F_{B}$ is the $B$-meson decay constant, defined in the usual way:

$\left\langle B(\vec{p})\left|J_{5, f}^{\mu}(x)\right| 0\right\rangle=-i F_{B} p^{\mu} \mathrm{e}^{i p x}$.

We have indicated with $|B(\vec{p})\rangle$ the state of a $B-$ meson with spatial momentum $\vec{p}$. This state is normalized in the following way:

$\langle B(\vec{p}) \mid B(\vec{q})\rangle=2 p_{B}^{0}(2 \pi)^{3} \delta^{(3)}(\vec{p}-\vec{q})$,

where $p_{B}^{0}=\sqrt{\vec{p}^{2}+M_{B}^{2}}$ is the energy.

Using the above-mentioned value of $M_{B}$ and the rough estimate $M_{b} \approx 5 \mathrm{GeV}$ for the mass of the $b$ quark, we derive:

$\mu_{\infty}=M_{B}-M_{b} \approx 300 \mathrm{MeV}$.

That is, after conversion to length units, $\lambda_{\infty} \equiv$ $1 / \mu_{\infty} \approx 0.66 \mathrm{fm}$. This value is in good agreement with the value $\lambda_{0} \simeq 0.63 \mathrm{fm}$ that we have obtained at the lightest quark mass $a \cdot m_{q}=0.01$ from a best fit with the function (3). An approach which uses the QCD sum-rules techniques gives the value $\mu_{\infty} \approx 290 \div 360 \mathrm{MeV}$ [10], which is in good agreement with the estimate (20) and with the above-mentioned lattice result.

\section{DISCUSSION AND CONCLUSIONS}

We have reconsidered the available lattice data for the gauge-invariant quark-antiquark nonlocal condensates [1]. A gaussian-type parametrization, inspired by the O.P.E. at short distances, fits well the data and gives results in agreement with the QCD sum-rules phenomenology. The original exponential-type parametrization fits also very well the data, in agreement with the expected large-distance behaviour of the correlators. The natural question which arises is: are the available lattice data for the correlators in the shortdistance or in the large-distance regime? It seems that they are just in an intermediate range. Further study is required in order to investigate more accurately both the short-distance and the largedistance asymptotic behaviour.

\section{Acknowledgements}

This work was supported by the EC TMR program ERBFMRX-CT97-0122. I would like to thank A. Di Giacomo and H.G. Dosch for many useful discussions.

\section{REFERENCES}

1. M. D'Elia, A. Di Giacomo and E. Meggiolaro, Phys. Rev. D 59 (1999) 054503.

2. S.V. Mikhailov and A.V. Radyushkin, JETP Lett. 43 (1986) 712; Sov. J. Nucl. Phys. 49 (1989) 494; Phys. Rev. D 45 (1992) 1754.

3. A.V. Radyushkin, Phys. Lett. B 271 (1991) 218.

4. A.P. Bakulev and A.V. Radyushkin, Phys. Lett. B 271 (1991) 223.

5. A.P. Bakulev and S.V. Mikhailov, Phys. Lett. B 436 (1998) 351.

6. A.E. Dorokhov, S.V. Esaibegyan and S.V. Mikhailov, Phys. Rev. D 56 (1997) 4062.

7. A.A. Pivoravov, Kratk. Soobshch. Fiz. (Bull. Lebedev Phys. Inst.) N 5 (1991) 3.

8. L.S. Brown and W.I. Weisberger, Phys. Rev. D 20 (1979) 3239.

9. E. Eichten and F. Feinberg, Phys. Rev. D 23 (1981) 2724.

10. H.G. Dosch and M. Eidemüller, private communication. 